\title{
Descripción clínica y socio-demográfica de pacientes psiquiátricos internados involuntariamente bajo el régimen de hospitalización administrativa en Chile Clinical and socio-demographic description of involuntary psychiatric inpatients admitted under the state of administrative hospitalization in Chile
}

\author{
Jaime Santander T. ${ }^{1}$, Daniela Huerta F. ${ }^{1}$, David Aceituno F. ${ }^{2}$ y Ximena Fuentes M. ${ }^{1}$
}

\begin{abstract}
Introduction: According to the "Operational framework for the admission of psychiatric inpatients and for the delivery of mental health services" in force since 2001, a person can be hospitalized at a psychiatric service without their agreement if their mental disorder poses a risk for their own safety or for the safety of other people. Aim: To describe the socio-demographic and clinical characteristics of psychiatric inpatients hospitalized under the "administrative hospitalization" rule (a specific type of involuntary hospitalization) at the psychiatric service of the Clinica San Carlos de Apoquindo between the years 2006 and 2008. Methods: Data was obtained from the patients clinical recordings. Results: Most of 32 patients were young women, active workers, with bipolar disorder and history of suicide behavior and poor medication compliance. Discussion: Our study is a first step to characterize the involuntary psychiatric inpatients population hospitalized under the procedures regulated by Chilean legislation established since less than ten years.
\end{abstract}

Key words: Involuntary commitment, patient admission, psychiatric department, psychiatry. Rev Chil Neuro-Psiquiat 2011; 49 (2): 157-164

\section{Introducción}

$\mathrm{L}$ a hospitalización por causa psiquiátrica ha generado desde sus orígenes debates en torno a su legitimidad toda vez que esta área de la medicina, como ninguna otra, enfrenta a pacientes que como corolario de sus trastornos no pueden acceder por libre voluntad a las herramientas diagnósticas ni terapéuticas que la internación en servicios especializados ofrece. Para los defensores acérrimos de las libertades civiles la hospitalización involuntaria de un paciente psiquiátrico es un atropello a sus libertades individuales, en cambio para los que enfrentan este problema desde una perspectiva médica, la hospitalización involuntaria de un paciente, que en ese momento no puede ejercer su

Recibido: 29/09/2011

Aprobado: 22/03/2011

\footnotetext{
Departamento de Psiquiatría, Facultad de Medicina, Pontificia Universidad Católica de Chile.
}

2 Interno de Medicina, Facultad de Medicina, Pontificia Universidad Católica de Chile. 
derecho de autonomía, es un acto de benevolencia hecho en el mejor interés del paciente con el fin de restablecer la competencia perdida ${ }^{1}$. Esta tensión tiene su expresión en el histórico debate entre los principios éticos de "autonomía" del paciente para tomar las decisiones pertinente acerca de su autocuidado, y el de "beneficencia", en que el equipo médico toma las decisiones para el cuidado del enfermos en consideración de las limitaciones transitorias o permanente que pueda tener un individuo para tomar una buena decisión informada. En el caso de la hospitalización contra voluntad se precisan circunstancias en que el equipo médico, y en definitiva, la sociedad a través del cuerpo legal existente, actúan, haciendo prevalecer el principio de beneficencia.

Durante la segunda mitad del siglo XX se alcanzaron acuerdos fundamentales en relación a los procedimientos que garantizan el resguardo de los derechos del paciente psiquiátrico que es hospitalizado involuntariamente. La Organización de Naciones Unidas con la promulgación en 1991 de los "Principios para la protección de los enfermos mentales y el mejoramiento de la atención de la Salud Mental"2 estableció estándares precisos para proteger a las personas con incapacidad mental. El principio 16 estipula que un paciente que padece una enfermedad mental puede ser hospitalizado involuntariamente sólo si a) debido a su enfermedad mental existe un riesgo grave de daño inminente para esa persona o terceros o b) que el hecho de no admitirlo puede provocar un deterioro considerable de su condición. La internación debe ser autorizada por un órgano de revisión independiente que cada estado determinará. Estos principios han sido utilizados por distintos países como modelo para formular legislaciones sobre salud mental.

En Chile, en 1998 fue aprobado mediante el decreto supremo $\mathrm{N}^{\circ} 570$ el "Reglamento para la internación de las personas con enfermedades mentales y sobre los establecimientos que la proporcionan", en vigencia desde enero de $2001^{3}$. En los artículos $8^{\circ}$ a $16^{\circ}$ de su título III, el Reglamento establece los tipos, condiciones y procedimientos para hospitalizar a personas con trastorno mental, entre los cuales se incluye la Internación Voluntaria y la Internación Involuntaria. La internación involuntaria puede ser de "urgencia" si dura menos de 72 horas o "administrativa" si dura más de 72 horas la que debe ser aprobada por la autoridad sanitaria, que desde el año 2005 es el Secretario Regional Ministerial de Salud (SEREMI). Es importante señalar que existe una tercera vía de internación contra voluntad, la llamada "judicial”, en la cual un juez puede determinar la internación para estudio y tratamiento de un procesado que así lo amerite. Por no tratarse de una situación que se origina en la práctica clínica no nos volveremos a referir a este tipo de internación.

El objetivo del presente trabajo es describir clínica y socio-demográficamente a un subgrupo de la población de pacientes psiquiátricos que han requerido ser internados bajo el régimen de hospitalización administrativa en el servicio de psiquiatría de la Clínica UC San Carlos de Apoquindo, con posterioridad a la entrada en vigencia del decreto supremo $\mathrm{N}^{\circ}$ 570. Esta información es importante por cuanto no existe a la fecha ningún reporte publicado acerca de cuál es el tipo de pacientes a quienes se aplica esta normativa ni de la magnitud de su utilización.

\section{Pacientes y Métodos}

\section{Reglamento de hospitalización involuntaria}

El "Reglamento para la internación de las personas con enfermedades mentales y sobre los establecimientos que la proporcionan" en su artículo 13 define a la Internación de Urgencia no Voluntaria como aquella requerida por las personas afectadas por enfermedad mental y que se encuentran en una crisis que conlleva riesgo inmediato para sí o para terceros y no aceptan voluntariamente la internación y que "sólo podrá extenderse por un período máximo de 72 horas". Esta internación debe ser resuelta por un médico, de preferencia especializado en psiquiatría, sin que en esta decisión se requiera la participación de la Autoridad Sanitaria.

Por otra parte, la Internación Administrativa (Artículo 14), es aquella que se determina por re- 
solución de la Autoridad Sanitaria competente, en las siguientes situaciones:

a) En el caso de una persona que fue hospitalizada involuntariamente de urgencia, que al cabo de 72 horas, continúa en crisis y persiste en no otorgar su consentimiento para continuar el tratamiento en régimen de internación.

b) En relación a una persona ha sido diagnosticada en la comunidad por un médico psiquiatra, como portadora de una crisis originada en una enfermedad mental, que se manifiesta por conductas que ponen en riesgo su integridad o la de los demás y no consiente la internación en forma voluntaria. La evaluación médica debe estar actualizada (al menos dentro de los últimos 30 días), efectuada por un médico psiquiatra de las redes asistenciales del Sistema Público o un psiquiatra del Sistema Privado.

La internación administrativa tiene una duración de 30 días. Si antes de este plazo el paciente completa un período de Internación Administrativa tal que permita alcanzar un estado mental clínicamente satisfactorio, para continuar su tratamiento con comprensión y autonomía suficientes, el médico tratante debe informar a la autoridad sanitaria a fin de que esta dicte la resolución de término de la Internación Administrativa, que puede dar paso a una internación voluntaria o seguimiento ambulatorio.

De mantenerse las condiciones que hicieron necesaria la Internación Administrativa más allá de 30 días, el paciente debe ser reevaluado por un médico psiquiatra distinto del tratante, para determinar la prórroga de la Internación Administrativa por períodos iguales y sucesivos si corresponde, dejando constancia en la ficha clínica de la persona y solicitando la prorroga a la Autoridad Sanitaria que ordenó la Internación Administrativa ${ }^{4}$.

\section{Recopilación de datos}

Se consideraron los datos de todos los pacientes que fueron hospitalizados entre los años 2006 y 2008 en el Servicio de Psiquiatría de la Clínica UC de la Pontificia Universidad Católica de Chile fueron recopilados desde los registros clínicos. Este servicio está dirigido a población que consulta a través de las modalidades de libre elección de los Institutos de Salud Provisional o en forma completamente privada. La recolección y utilización anónima de la información ahí contenida fue autorizada por el Comité de Ética de la misma universidad. Se estudiaron los antecedentes de todos los pacientes a los que se les realizó formalmente el trámite descrito de internación administrativa en el período de estudio. Los factores socio-demográficos incluidos fueron: edad, sexo, estado civil (soltero/ casado/separado), años de educación, ocupación (estudiante/ trabajador activo/ cesante) y red social (vive solo/ vive acompañado). Los antecedentes clínicos considerados fueron: diagnóstico psiquiátrico EJE 1 según el DSM-4 ${ }^{5}$ antecedente de intento suicida previo (sí/no), disfunción familiar (sí/no), adherencia a tratamiento (buena/mala/ otra) y duración de la hospitalización (en días). Se debe señalar que en los casos de comorbilidad se consignó la patología principal que motivó el ingreso e internación contra voluntad.

\section{Análisis estadístico}

Los datos fueron analizados usando el programa MINITAB 15 Statistical Software. Las variables categóricas (sexo, estado civil, red social, diagnóstico, intento suicida, disfunción familiar, ocupación, adherencia a tratamiento) fueron expresadas en términos porcentuales. Las variables numéricas (edad, años de educación, días de estadía) dado el tamaño de la muestra fueron expresadas en términos de mediana y rango inter-cuartil (25 y 75\%).

\section{Resultados}

Durante el período observado 1.086 pacientes fueron hospitalizados en el servicio, 32 (2,94\%) de los cuales lo hicieron bajo el régimen de hospitalización administrativa. La Tabla 1 resume la caracterización socio-demográfica de la muestra según las variables categóricas evaluadas. La mayor parte de los pacientes hospitalizados involuntariamente fueron mujeres $(62,5 \% ; \mathrm{n}=20)$, solteros (as) $(62,5 \% ; \mathrm{n}=20)$ con trabajo activo al momento de la hospitalización $(59,38 \% ; \mathrm{n}=19)$ y compartien- 
do habitación con al menos una persona $(68,75 \%$; $\mathrm{n}=22$ ). La Tabla 2 resume la caracterización clínica de la muestra según las variables categóricas. La patología psiquiátrica de base mayormente reportada fue "Trastorno Bipolar" $(50 \% ; n=16)$ seguido de "Abuso de sustancias" (21,88\%; $n=7)$ finalizando con "Esquizofrenia" y "Depresión Unipolar" ambas con un $12,5 \%(n=4)$. La mayor parte de los pacientes tenía antecedente de intento suicida previo a la hospitalización $(75 \% ; \mathrm{n}=24)$, sin intento suicida en la hospitalización actual $(87,5 \% ; \mathrm{n}=28)$ mala adherencia al tratamiento $(78,13 \% ; \mathrm{n}=25) \mathrm{y}$ disfunción familiar $(59,38 \% ; \mathrm{n}=19)$.

La Tabla 3 resume la caracterización de la muestra según las variables numéricas evaluadas. La mediana de edad observada fue de 34 años con un rango inter-cuartil (RIC) entre 27 y 48 años. La mediana de años de estudio fue de 16 años (RIC 12-17). La mediana de días de hospitalización correspondió a 25 días (RIC 16-31).

Tabla 1. Descripción características socio-demográficas 32 pacientes hospitalizados administrativamente entre los años 2006 y 2008 según sexo, estado civil, red social y ocupación, diagnóstico, intento suicida, síntomas psicóticos, disfunción familiar y adherencia a tratamiento

\begin{tabular}{|lcc|}
\hline Características & n & $\%$ \\
Socio-demográficas & & \\
Sexo & 20 & 62,5 \\
Masculino & 12 & 37,5 \\
Estado Civil & & \\
Soltero (a) & 20 & 62,5 \\
Casado (a) & 6 & 18,7 \\
Separado/divorciado (a) & 6 & 18,7 \\
Viudo & 0 & 0 \\
Ocupación & & \\
Estudiante & 5 & 15,6 \\
Trabajador activo & 19 & 59,4 \\
Cesante & 8 & 25,0 \\
Red social & & \\
Vive solo & 10 & 31,3 \\
Vive acompañado & 22 & 68,7 \\
\hline
\end{tabular}

Tabla 2. Descripción características clínicas de 32 pacientes hospitalizados administrativamente entre los años 2006 y 2008 según diagnóstico, intento suicida (previo y actual), síntomas psicóticos, disfunción familiar $y$ adherencia a tratamiento

\begin{tabular}{|lcc|}
\hline Características clínicas & n & $\%$ \\
Diagnóstico & & \\
Depresión unipolar & 4 & 12,5 \\
Trastorno bipolar & 16 & 50,0 \\
Esquizofrenia & 4 & 12,5 \\
Abuso de sustancias & 7 & 21,8 \\
Otros & 1 & 3,1 \\
Síntomas psicóticos & & \\
Sí & 12 & 37,5 \\
No & 20 & 62,5 \\
Intento Suicida & & \\
(previo a la hospitalización) & & \\
Sí & 24 & 75,0 \\
No & 8 & 25,0 \\
Intento Suicida & & \\
(en ingreso actual) & 25,3 \\
Sí & & \\
No & & \\
Adherencia a tratamiento & & \\
Buena & & \\
Mala & 28,5 \\
Disfunción familiar & & \\
\hline No & & \\
\hline
\end{tabular}

Tabla 3. Descripción características socio-demográficas y clínicas de los 32 pacientes hospitalizados administrativamente entre los años 2006 y 2008 según edad, años de estudio y días de estadía

\begin{tabular}{|lcccc|}
\hline Características & $\mathbf{n}$ & Mediana & $\mathbf{2 5 \%}$ & $\mathbf{7 5 \%}$ \\
Edad & 32 & 34 & 27 & 48 \\
Años de estudio & 32 & 16 & 12 & 17 \\
Días de estadía & 32 & 25 & 16 & 31 \\
\hline
\end{tabular}




\section{Discusión}

Nuestro objetivo fue describir las características clínicas y socio-demográficas de todos los pacientes hospitalizados involuntariamente bajo el régimen de internación administrativa en el servicio de psiquiatría de la Clínica UC San Carlos de Apoquindo entre los años 2006 y 2008. Treinta y dos pacientes fueron hospitalizados administrativamente correspondiendo a un 2,94\% del total de pacientes hospitalizados durante ese período. Las cifras de prevalencia de hospitalizaciones involuntarias reportadas en la literatura son heterogéneas, con un rango entre 2 y $36 \%{ }^{6,7}$. Esta variabilidad puede ser explicada por múltiples factores, entre ellos la definición que cada país tiene del término hospitalización involuntaria así como también la existencia de normativas que regulen este tipo de internaciones. Otro factor importante a considerar es el tipo de hospitalización involuntaria que se incluye en cada estudio. En general, las distintas legislaciones que regulan estos procedimientos definen un período de "ventana" entre el ingreso involuntario del paciente y la solicitud de aprobación de esta misma a la autoridad correspondiente, que comúnmente dura entre 48 a 72 horas (definido en nuestro reglamento como hospitalización involuntaria de urgencia). Durante este período el paciente puede: a) ser dado de alta; b) consentir su internación o c) mantener su negativa a ser hospitalizado aún cuando los síntomas que motivaron su internación persisten. Este último caso, generará la solicitud de aprobación a la autoridad para mantener la hospitalización (en nuestro país hospitalización administrativa). Ha sido demostrado que hasta un $47 \%$ de los pacientes que ingresan involuntariamente consienten su hospitalización durante este período de ventana ${ }^{8}$, por lo que, finalmente el número de hospitalizaciones que requieren aprobación por parte de la autoridad sanitaria decrece, lo que se refleja en prevalencias disímiles dependiendo del tipo de hospitalización involuntaria incluida en cada estudio.

\section{Variables socio-demográficas}

La mayor proporción de mujeres observada $(62,5 \%)$ difiere de lo reportado habitualmente en la literatura internacional ${ }^{9,10}$ que sitúa el sexo masculino como un factor fuertemente asociado a la internación involuntaria por causa psiquiátrica. La proporción total de mujeres hospitalizadas durante los años 2006 a 2008 en nuestro servicio corresponde a un $62,15 \%(n=675)$, es decir, nuestra población se compone fundamentalmente de mujeres, lo que contribuye a explicar parcialmente que este sexo sea el más representado en las hospitalizaciones involuntarias.

En relación al resto de las variables socio-demográficas analizadas, la mayor parte de la muestra correspondió a pacientes solteros, adultos jóvenes y viviendo acompañados al momento de la hospitalización lo que concuerda con reportes previos ${ }^{9,10}$. La mediana de años de educación observada en nuestro estudio (16 años) es mayor a lo que muestran estudios previos (12 años o menos $)^{6,10}$. Asimismo, nuestra población estuvo conformada fundamentalmente de personas que trabajaban activamente al momento de la hospitalización lo que ha sido escasamente señalado con anterioridad. Nosotros especulamos que estos últimos resultados pueden relacionarse al estrato económico al que nuestra muestra pertenece (estrato económico alto), el que se asocia a más años de estudio y mayores oportunidades laborales.

\section{Variables clínicas}

La mayor parte de los pacientes hospitalizados involuntariamente tenían como diagnóstico primario el "Trastorno Bipolar" (50\%; $\mathrm{n}=16)$, esto difiere de lo habitualmente señalado en la literatura que reporta a la Esquizofrenia como el diagnóstico psiquiátrico que más se asocia a la hospitalización involuntaria. Sin embargo, pocos estudios han evaluado la distribución de los diagnósticos según sexo. Entre ellos destaca el estudio de Sanguineti et $\mathrm{al}^{11}$, quien a partir de una muestra de 815 pacientes esquizofrénicos hospitalizados involuntariamente mostró que la mayor proporción de ellos fueron hombres. Por otro lado, Schuepbach et $\mathrm{al}^{12}$, basado en una muestra de 55 pacientes con diagnóstico de Trastorno Bipolar hospitalizados involuntariamente, reportó que la mayor parte de ellos fueron mu- 
jeres. Esto podría explicar en parte que, dado que nuestra muestra se compone fundamentalmente de mujeres, el "Trastorno Bipolar” sea la patología más frecuente toda vez que el diagnóstico psiquiátrico asociado a la hospitalización involuntaria dependería del sexo del paciente.

Con respecto al resto de variables clínicas evaluadas, observamos una alta proporción pacientes con mala adherencia a tratamiento así como con disfunción familiar, resultados que concuerdan con lo reportado previamente en la literatura ${ }^{8,13}$. Una observación interesante fue la baja proporción de pacientes que presentaron síntomas psicóticos, factor que ha sido ampliamente asociado a este tipo de hospitalización ${ }^{8,14}$, lo que podría ser explicado debido a la relativamente baja frecuencia de esquizofrenia en nuestra muestra.

En relación al comportamiento suicida de nuestros pacientes, es relevante señalar que la mayor parte de ellos presenta antecedentes de suicidio previo, sin embargo, sólo una pequeña fracción exhibe este comportamiento en el episodio que origina la actual hospitalización. Lo anterior podría evidenciar una tendencia a "prevenir" la comisión de suicidio en pacientes con historia de autoagresión a través de la hospitalización, toda vez que sabemos que el antecedente de intento de suicidio previo es uno de los factores de riesgo mayor para su comisión efectiva ${ }^{15,16}$.

Mayoritariamente, los pacientes permanecieron hospitalizados alrededor de 25 días (RIC: 15-31), es decir, la mayor parte de los pacientes mostraron mejoría antes del término del plazo autorizado siendo dados de alta para continuar tratamiento en forma ambulatoria.

\section{Limitaciones y futuras perspectivas}

Nuestro estudio adolece de algunas limitaciones. En primer lugar, la naturaleza observacional y descriptiva del estudio obliga a que en una siguiente etapa, diseñemos un estudio de cohorte que permita establecer las diferencias demográficas y clínicas entre los pacientes que se hospitalizan involuntaria $v s$ voluntariamente. Por otra parte, esta investigación fue desarrollada en el Servicio de
Psiquiatría de la Clínica San Carlos de Apoquindo, centro privado que atiende a una población de estrato económico alto, con indicación de hospitalización aguda (no es un establecimiento de tratamiento institucional de pacientes crónicos), por lo tanto, nuestros resultados no representan necesariamente las características que observaríamos en la población general de pacientes hospitalizados involuntariamente en Chile. Será interesante comparar estos hallazgos con estudios futuros realizados en otros servicios psiquiátricos del país, con otro tipo de población y en otros contextos sociales.

\section{Conclusiones}

En nuestro conocimiento, sólo un estudio chileno fue dirigido previamente con el fin de evaluar las características de pacientes psiquiátricos internados involuntariamente ${ }^{17}$ realizado con anterioridad a la entrada en vigencia del decreto $\mathrm{N}^{\circ} 570$. Nuestro estudio es, por lo tanto, el primer trabajo destinado a describir a la población psiquiátrica hospitalizada involuntariamente posterior a la entrada en vigencia de dicho decreto. Nuestro servicio desde sus orígenes implementa este reglamento, llevando registro de cada uno de los pacientes que fueron sometidos a este procedimiento lo que nos permitió esbozar un perfil tanto socio-demográfico como clínico de ellos. En relación al perfil sociodemográfico, observamos que la mayor parte de la muestra correspondió a un paciente de sexo femenino, adulto joven, trabajando activamente, viviendo con al menos una persona más y con un alto nivel educacional. El perfil clínico corresponde a un paciente con trastorno bipolar, que presenta antecedentes de intentos de suicidio, mala adherencia a tratamiento y conflictos familiares, lo que probablemente refleja ausencia de conciencia de enfermedad previa, validando el espíritu del decreto 570 que intenta proteger al paciente y a su familia en caso de crisis por descompensación de su patología.

Nuestro estudio, establece las bases para el desarrollo de investigaciones que extiendan nuestros resultados con el objeto de determinar factores que 
aumenten el riesgo de hospitalizar a una persona involuntariamente por causa psiquiátrica, con el fin de generar a largo plazo estrategias de preven- ción efectivas y racionales que ayuden a favorecer el manejo ambulatorio de estos pacientes como lo recomiendan organismos internacionales.

\begin{abstract}
Resumen
Introducción: El "Reglamento para la internación de las personas con enfermedades mentales y sobre los establecimientos que la proporcionan" en vigencia desde 2001 establece que una persona sólo puede ser hospitalizada en un servicio psiquiátrico sin su consentimiento si como consecuencia de su patología psiquiátrica pone en riesgo su seguridad o la de terceros. Objetivo: Describir socio-demográfica y clínicamente a pacientes que fueron internados bajo el régimen de hospitalización administrativa (caso especial de la hospitalización involuntaria) en el servicio de psiquiatría de la Clínica San Carlos de Apoquindo. Métodos: Se revisó retrospectivamente los registros clínicos de todos los pacientes que fueron hospitalizados involuntariamente entre los años 2006 y 2008. Resultados: Se describen 32 casos, principalmente mujeres jóvenes con trabajo activo al momento de la hospitalización, con diagnóstico de trastorno bipolar y con historia de intentos de suicidio previos y mala adherencia al tratamiento. Discusión: El presente estudio representa un avance en la caracterización de la población de pacientes psiquiátricos que se hospitalizan involuntariamente dentro del marco legal establecido en Chile hace menos de una década.

Palabras clave: Compromiso involuntario, admisión de paciente, departamento psiquiátrico, psiquiatría.
\end{abstract}

\section{Referencias}

1. Chodoff P. Involuntary hospitalization of the mentally ill as a moral issue. Am J Psychiatry 1984; 141 (3): 384-9.

2. Principles for the protection of persons with mental illness and the improvement of mental health care. In: UN, editor. GA Res 119: UN GAOR; 1991.

3. República de Chile Ministerio de Salud, Departamento de Asesoría Jurídica. Reglamento para la internación de las personas con enfermedades mentales y sobre los establecimientos que la proporcionan. In: República de Chile, Ministerio de Salud. DTON570/98. D.OF. 14.07.00; 2000.

4. Gobierno de Chile Ministerio de Salud. Circular A15/Nº13. Santiago; 2007.

5. Association AP. Diagnostic and statistical manual of mental disorders: DSM-IV. Washington: American Psychiatric Association; 1994.
6. Potkonjak J, Karlovic D. Sociodemographic and medical characteristics of involuntary psychiatric inpatients--retrospective study of five-year experience with Croatian Act on Mental Health. Acta Clin Croat 2008; 47 (3): 141-7.

7. Salize HJ, Dressing H. Epidemiology of involuntary placement of mentally ill people across the European Union. Br J Psychiatry 2004; 184: 163-8.

8. Craw J, Compton MT. Characteristics associated with involuntary versus voluntary legal status at admission and discharge among psychiatric inpatients. Soc Psychiatry Psychiatr Epidemiol 2006; 41 (12): 981-8.

9. Sanguineti VR, Samuel SE, Schwartz SL, Robeson MR. Retrospective study of 2,200 involuntary psychiatric admissions and readmissions. Am J Psychiatry 1996; 153 (3): 392-6.

10. Bauer A, Rosca P, Grinshpoon A, Khawaled R, Mester R, Yoffe R, et al. Trends in involuntary 
psychiatric hospitalization in Israel 1991-2000. Int J Law Psychiatry 2007; 30 (1): 60-70.

11. Sanguineti VR, Samuel SE, Schwartz SL, Robeson MR. Gender differences among civilly committed schizophrenia subjects. Schizophr Bull 1996; 22 (4): 653-8.

12. Schuepbach D, Goetz I, Boeker H, Hell D. Voluntary vs involuntary hospital admission in acute mania of bipolar disorder: results from the Swiss sample of the EMBLEM study. J Affect Disord 2006; 90 (1): 57-61.

13. Ishizuka C, Nakatani Y, Morita N, Satoh S. Sociodemographic and clinical characteristics of psychiatric patients coercively brought to hospitals. Psychiatry Clin Neurosci 2001; 55 (2): 147-56.
14. Kozumplik O, Jukic V, Goreta M. Involuntary hospitalizations of patients with mental disorders in Vrapce Psychiatric Hospital: five years of implementation of the first Croatian law on protection of persons with mental disorders. Croat Med J 2003; 44 (5): 601-5.

15. Hawton K, van Heeringen K. Suicide. Lancet 2009; 373 (9672): 1372-81.

16. Yoshimasu K, Kiyohara C, Miyashita K. Suicidal risk factors and completed suicide: meta-analyses based on psychological autopsy studies. Environ Health Prev Med 2008; 13 (5): 243-56.

17. Bahamondes A. Hospitalizaciones forzosas en psiquiatría. Revista Chilena de Derecho 1995; 22 (2): 7 .

Correspondencia:

Jaime Santander Toro

Departamento de Psiquiatría

Escuela de Medicina

Pontificia Universidad Católica de Chile

Apoquindo 3990 - Of. 502

Las Condes, Santiago.

Fax: 56-2-2070583

E-mail: jsantan@med.puc.cl 